\title{
Associations of maternal upper respiratory tract infection/influenza during early pregnancy with congenital heart disease in offspring: evidence from a case-control study and meta-analysis
}

\author{
Y. Q. Xia ${ }^{1+}$, K. N. Zhao ${ }^{1+}$, A. D. Zhao', J. Z. Zhu², H. F. Hong ${ }^{3}$, Y. L. Wang ${ }^{4^{*}}$ and S. H. Li ${ }^{1 *}$ (D)
}

\begin{abstract}
Background: Evidences regarding the associations between maternal upper respiratory tract infection/influenza during pregnancy and the risk of congenital heart disease (CHD) is still controversial. This study was specifically designed to examine the associations by a case-control study and a meta-analysis of the published evidences and our finding.

Methods: A hospital-based case-control study involving 262 children with simple CHD and 262 children with complex CHD, along with 262 control children, was conducted through June, 2016 to December, 2017. All children were aged 0-2 years old. Furthermore, a meta-analysis based on both previously published studies and our case-control study was performed.

Results: In the case-control study, after adjusting for possible confounders, maternal upper respiratory tract infection/ influenza during early pregnancy was found to be related to an increased risk of CHD (OR $=3.40$ and 95\% Cl: 2.05-5.62 for simple CHD; $\mathrm{OR}=2.39$ and $95 \% \mathrm{Cl}: 1.47-3.88$ for complex $\mathrm{CHD}$ ). After a meta-analysis, the adverse impact was still kept significant $(\mathrm{OR}=1.47$ and $95 \% \mathrm{Cl}: 1.28-1.67$ for simple $\mathrm{CHD} ; \mathrm{OR}=1.44$ and $95 \% \mathrm{Cl}: 1.14-1.75$ for complex CHD). The very similar associations were also observed among single type of CHD, herein, ventricular septal defects (VSD) and tetralogy of fallot (TOF) in the case-control study. In the subsequent meta-analysis, however, the significant association only existed in VSD.
\end{abstract}

Conclusions: Although there is still conflicting in TOF, the results are overall consistent, which provide new enforced evidence that maternal upper respiratory tract infection/influenza during early pregnancy, in general, play an important role in the occurrence of $\mathrm{CHD}$.

Keywords: Early pregnancy, Upper respiratory tract infection, Influenza, Congenital heart disease

\footnotetext{
*Correspondence: wyanlin@163.com; Ish9907@163.com

${ }^{\dagger}$ Y. Q. Xia and K. N. Zhao contributed equally to this work.

${ }^{4}$ Prenatal Diagnosis Department, International Peace Maternity \& Child Health

Hospital, Shanghai Jiao Tong University School of Medicine, 910 Hengshan

Road, Xuhui District, Shanghai 200030, China

'School of Public Health, Shanghai Jiao Tong University, 227 South

Chongqing Road, Huangpu District, Shanghai 200025, China

Full list of author information is available at the end of the article
}

(c) The Author(s). 2019 Open Access This article is distributed under the terms of the Creative Commons Attribution 4.0 International License (http://creativecommons.org/licenses/by/4.0/), which permits unrestricted use, distribution, and reproduction in any medium, provided you give appropriate credit to the original author(s) and the source, provide a link to the Creative Commons license, and indicate if changes were made. The Creative Commons Public Domain Dedication waiver (http://creativecommons.org/publicdomain/zero/1.0/) applies to the data made available in this article, unless otherwise stated. 


\section{Background}

Congenital heart disease (CHD) has been the most prevalent birth defect [1], and it remains to be an important cause of neonatal and infant deaths [2, 3]. It was reported that the global incidence of $\mathrm{CHD}$ had increased nearly 15 times over the past approximately 60 years, from $0.6 \%$ in $1930-1934$ to $9.1 \%$ in 1995 [1]. In China, the rising trend similarly existed, and the prevalence of CHD among newborns had reached up to $11.1 \%$ [4]. Therefore, CHD has become a severe public health challenge all around the world.

The exact cause of CHD is still unclear. Generally, only $10-15 \%$ of CHD could be explained by gene defects and chromosome abnormalities, and the majority should be caused by the interaction of genetic factors and environmental exposures [5]. Early pregnancy is a key period for the development of fetal cardiovascular system, especially 14-60 days of gestation is the vulnerable window for CHD [6]. Upon the perspective of prevention, it is critically important to explore and identify CHD-related risk factors, especially those factors that can be intervened.

Upper respiratory tract infection and influenza always outbreak seasonally, in which season pregnant women are susceptible to the infection [7-9]. Previous studies have explored the relationship between maternal upper respiratory tract infection during pregnancy and the risk of CHD [10-25]. A number of evidence seemed to support the association [11, 16, 19, 20, 22, 23], however, a few other studies did not establish the relationship $[12,13]$. Similarly, findings on the association between influenza during pregnancy and CHD was also controversial $[11,13-15,18-21,24,25]$. Almost all these studies took all CHD types as a whole. If the role of maternal upper respiratory tract infection/influenza varies in different type of CHD, then the possible different CHD type constitution may, at least partly, explains the inconsistent finding.

The present study aimed at exploring the associations of maternal upper respiratory tract infection/influenza during early pregnancy with CHD through a casecontrol study and a further meta-analysis. We observed if the associations were different between simple and complex CHD. Meanwhile, we paid particular attention to ventricular septal defects (VSD) and tetralogy of fallot (TOF), the most prevalent type of simple and complex CHD, respectively.

\section{Methods}

Hospital-based case-control study

Subjects

A hospital-based case-control study was conducted in Shanghai Children's Medical Center through June, 2016 to December, 2017. Detailed study methods have been published elsewhere [26]. Briefly, study sample consisted of 524 cases (262 children with simple CHD and 262 children with complex CHD) and 262 controls without any birth defects. Among the controls, 132 was enrolled from pediatric respiratory medicine, 91 from pediatric general surgery, and 39 from pediatric gastroenterology. All the children were younger than 2 years old, and were recruited during the same period.

CHD was defined based on clinical diagnosis and verification by ultrasound. According to the codes of the International Classification of Diseases, Tenth Revision, Clinical Modification, complex CHD refers to all types of CHD except for simple CHD. The main types of simple CHD include VSD, secundum atrial septal defects, coarctation of aorta, patent ductus arteriosus, pulmonary stenosis, aortic valve stenosis, pulmonary stenosis, and etc. The main types of complex CHD include TOF, transposition of great arteries, double outlet right ventricle, hypoplastic left heart syndrome, tricuspid atresia and stenosis, and etc. $[27,28]$.

\section{Ethical approval}

The study conforms to the declaration of Helsinki, and the ethical application and consent procedure were approved by the Ethics Committee of Shanghai Jiao Tong University School of Medicine (Approval number: SJUPN201717). Written informed consent was received from all participants prior to inclusion.

\section{Associated factors of CHD}

Information on sociodemographic characteristics and parental health-related behaviors and characteristics was retrospectively collected through the Parental Behaviors and Environmental Exposure Questionnaire (PBEQ), which has been used in our previous study [26]. The women who signed informed consent were invited to participate in an interview, and to fill in the PBEQ. Maternal upper respiratory tract infection/influenza exposure during early pregnancy was evaluated by two questions in the PBEQ. One is to ask: "Did you ever have upper respiratory infection (cold) and/or influenza during pregnancy?", and the response was "yes" or "no". Another one is a multiple-choice question regarding the period: "In which period of gestation did you get upper respiratory infection (cold) and/or influenza? The response is shown as: 1 for early pregnancy, 2 for second trimester, 3 for the third trimester.

Demographic and obstetric characteristics There are 9 variables included parental ethnic, maternal age at delivery, maternal educational level, marital status, maternal residence, maternal prepregnancy obesity, maternal multiple births, infant gender and family history. The 
specific classification method of variables have previously been published [26].

Maternal health indicators during pregnancy Maternal prepregnancy diabetes/hypertension (no/yes); maternal folic acid use (no/yes); and maternal smoking/ drinking (defined as maternal previous history of smoking and/or drinking; no/yes).

\section{Statistical analyses}

Statistical descriptions including the number and percentage for categorical variables, and Chi-squared test was used to compare group difference. Logistic regression analyses were further applied to examine the crude and adjusted associations of maternal upper respiratory tract infection/influenza during early pregnancy with CHD. A two-step procedure was implemented for adjustments. Maternal ethic, maternal age at delivery, maternal education, marital status, residence, maternal prepregnancy obesity, multiple births, infant gender, and family history of CHD were adjusted in model I. In model II, prepregnancy diabetes/hypertension, folic acid use, smoking/drinking, were simultaneously further controlled. All the analyses were also repeated after controlling for the potential selection bias between cases and controls through propensity score matching, in which propensity score was calculated based on all covariates mentioned above. Propensity score was estimated by multivariable logistic regression model, in which all potential confounding variables related to CHD were included. The greedy nearest neighbor matching propensity score algorithm was applied to propensity score matching. Match the control with the case using caliper 0.1 . R statistics software was utilized with Matchit software package.

All the analyses were performed with the Statistical Package for the Social Sciences (SPSS) (IBM-SPSS Statistics v22.0, Inc. Chicago, IL). A statistical significance level was set at a 2 -tail $p$-value $<0.05$.

\section{Meta-analysis \\ Literature search}

We, furthermore, conducted a meta-analysis of all previous published studies and our case-control study on the associations between maternal upper respiratory tract infection/influenza and CHD. The relevant studies published up to Nov 4, 2018 were retrieved in the Pubmed, Embase, and Cochrane, without any language restriction, using the following terms: ('pregnancy' or 'pregnant women' or 'mothers' or 'gravidity' or 'gestation') and ('heart defects, congenital' or 'congenital heart defects' or 'congenital heart disease' or 'heart abnormalities' or 'abnormalities, heart' or 'heart, malformation of') and ('respiratory tract infections' or 'infection, respiratory tract' or 'upper respiratory tract infections' or 'respiratory infection' or 'infections, respiratory' or 'common cold' or 'cold, common' or 'acute coryza' or 'coryza, acute' or 'influenza, human' or 'influenza' or 'flu' or 'grippe') (the search strategy in Embase is shown in Additional file 1). Bibliographies of retrieved articles were also reviewed to identify additional eligible articles.

\section{Inclusion and exclusion criteria}

The retrieved literatures were included in the metaanalysis when they met all the following inclusion criteria: 1) Observational studies without any intervention that focused on pregnant women; 2) Outcome: The diagnosis of CHD in offspring was clearly presented and the diagnostic approach of cases and control groups was identical; 3) Exposure: Risk factors for CHD included maternal upper respiratory tract infection and/or influenza exposure during pregnancy; 4) Data: The information of odd ratio (OR) and 95\% confidence interval (95\% CI) could be obtained. As for the studies from the same researchers, the one with larger sample size would be included since the precision was driven primarily by the sample size.

\section{Data extraction}

For each included study, we extracted information as follows: name of author, publication year, country of origin, study type, sample size, type of CHD, maternal exposure including upper respiratory tract infection and influenza, and ORs (95\% CIs). All data from original studies were extracted independently by two authors and compared afterwards. In cases of conflicting evaluations, any discrepancy would be resolved by a third reviewer.

\section{Statistical analyses}

ORs, along with corresponding 95\% CIs, were applied to assess the associations of maternal upper respiratory tract infection/influenza during early pregnancy with CHD in offspring. Heterogeneity was determined with Cochran's Q-statistic (significance level was set at $p<$ 0.1 ), and was quantified with $\mathrm{I}^{2}$ test, in which $\mathrm{I}^{2}>50 \%$ could be regarded as the moderate-to-high heterogeneity [29]. Random-effect model (DerSimonian-Laird method) was used if heterogeneity between studies was present; otherwise, a fixed-effect model (Mantel-Haenszel method) should be applied [30]. Additionally, the impact of individual studies on our meta-analysis summary statistic was evaluated by sensitivity analysis, which removes each study. In order to make our analysis more reliable, we compared the reappraised results with original results. Finally, publication bias was assessed by Begg's test and Egger's linear regression test, and the effect on the interpretation of results was estimated by the trim-and- 
fill computation [31]. All the statistical analyses were conducted using STATA version 14.0 (Stata Corp, College Station, TX, USA).

\section{Results}

\section{Hospital-based case-control study}

Among 262 children with simple CHD in this study, VSD was the most prevalent form (63.4\%), and then atrial septal defects (51.9\%) and patent ductus arteriosus (14.9\%). For 262 children with complex CHD, TOF accounted the most (37.0\%), and then transposition of great arteries (10.7\%) and single ventricle (6.9\%).

The sample characteristic was presented in Table 1. It could be seen that maternal folic acid use, maternal residence and maternal education level were significantly different between almost all CHD groups vs. Controls (all $p<0.05$ ). In addition, infant gender and family history of CHD were also different, however, mainly between simple CHD and control (both $p<0.05$ ), and the difference of smoking/drinking only existed between TOF and controls $(p<0.05)$. After propensity score matching, all these covariates were well balanced (all $p>$ 0.05) (Additional file 2: Table S1).

The associations of maternal upper respiratory tract infection/influenza during early pregnancy with CHD in offspring were shown in Table 2. It was found that maternal upper respiratory tract infection/influenza during early pregnancy was a significant risk factor for both simple and complex CHD in univariate regression models. Through a two-step controlling procedure in multivariate regression models, the significant findings still remained. In the final full models, after adjusting for both demographic and obstetric characteristics and maternal health indicators during pregnancy, maternal upper respiratory tract infection/influenza during early pregnancy, compared to controls, could increase the risk of simple and complex CHD by $2.43(\mathrm{OR}=3.43,95 \% \mathrm{CI}$ : 2.12-5.54) and 1.43 times ( $\mathrm{OR}=2.43$, 95\% CI: $1.54-$ 4.45), respectively. Particularly, we also observed the similar associations in VSD $(\mathrm{OR}=3.99,95 \% \mathrm{CI}$ : $1.94-$ 8.18) and TOF (OR $=2.93,95 \% \mathrm{CI}: 1.56-5.52)$. When the analyses were repeated after propensity score matching, similar results were obtained (Additional file 2: Table S2).

\section{Meta-analysis}

The flow chart indicates the study process of literature selection and exclusion/inclusion criteria in the metaanalysis (Fig. 1). The 16 studies, including 11,911 cases and 74,358 controls, were finally identified as eligible in the analyses [10-25], covering a period from 1989 to 2018. All the 16 included studies were case-control studies. Among them, four only focused on maternal upper respiratory tract infection/influenza and the risk of $\mathrm{CHD}$ and the others included not only maternal upper respiratory tract infection/influenza but also other risks.

As for maternal upper respiratory tract infection/influenza and CHD, the random effects model was used since between-study heterogeneity existed $\left(I^{2}=37.3 \%, \quad p=\right.$ 0.056). The pooled analysis of the 16 studies, together with our study presented here, was performed and revealed significant associations with OR $(95 \% \mathrm{CI})$ of 1.43 (1.24-1.63) (Fig. 2). Furthermore, the CHD was divided into two subgroups as simple CHD and complex CHD, and the two fixed effects models were applied since no significant between-study heterogeneity for simple CHD $\left(I^{2}=26.6 \%, p=0.133\right)$ and complex CHD $\left(I^{2}=0.0 \%, p=\right.$ $0.520)$ was found. Similarly, results of the pooled analyses showed that maternal upper respiratory tract infection/influenza during early pregnancy was associated with both simple $\mathrm{CHD}(\mathrm{OR}=1.47,95 \% \mathrm{CI}: 1.28-1.67)$ (Fig. 3) and complex CHD (OR $=1.44$, 95\% CI: $1.14-$ 1.75) (Fig. 4). The comprehensive results showed that maternal upper respiratory tract infection/influenza had different effect sizes on VSD (OR $=1.34,95 \%$ CI: $1.16-$ 1.51) (Fig. 5) and TOF (OR $=0.96,95 \%$ CI: 0.42-1.50) (Fig. 6). All the pooled analyses were also repeated based on our results after propensity score matching and the same 16 published studies, the associations were largely unchanged (Additional file 2: Figures S1-S5).

To examine the impact of individual study on the overall results, sensitivity analyses were carried out (Additional file 2: Figures S6-S10). The pooled ORs of maternal upper respiratory tract infection/influenza with CHD were not affected by the results of any individual study, suggesting the statistically robust results of this meta-analysis. Furthermore, publication bias was evaluated by performing Begg's test and Egger's linear regression test (statistical significance was set at $p<0.10$ ). Neither Begg's test nor Egger's test found significant publication biases either in subgroup of simple CHD $(\mathrm{z}=0.49, p=0.626 ; \mathrm{t}=0.29, p=0.776)$ or in complex $\mathrm{CHD}(\mathrm{z}=0.50, p=0.620 ; \mathrm{t}=-0.33, p=0.743)$. However, publication biases existed in all CHD as a whole group $(\mathrm{t}=2.17, p=0.046$ in Egger's test) (Additional file 2: Table S3). However, the further analysis with trim-andfill test indicated that this level of publication bias did not impact the estimates (ie, no trimming done because data unchanged) [32].

\section{Discussion}

Up to now, this is the largest population-based epidemiology study $(n=86,269)$ exploring the relationship between maternal upper respiratory tract infection/ influenza and the risk of CHD in offspring. In this study, we examined the associations through a case-control study and a further meta-analysis based on previous 


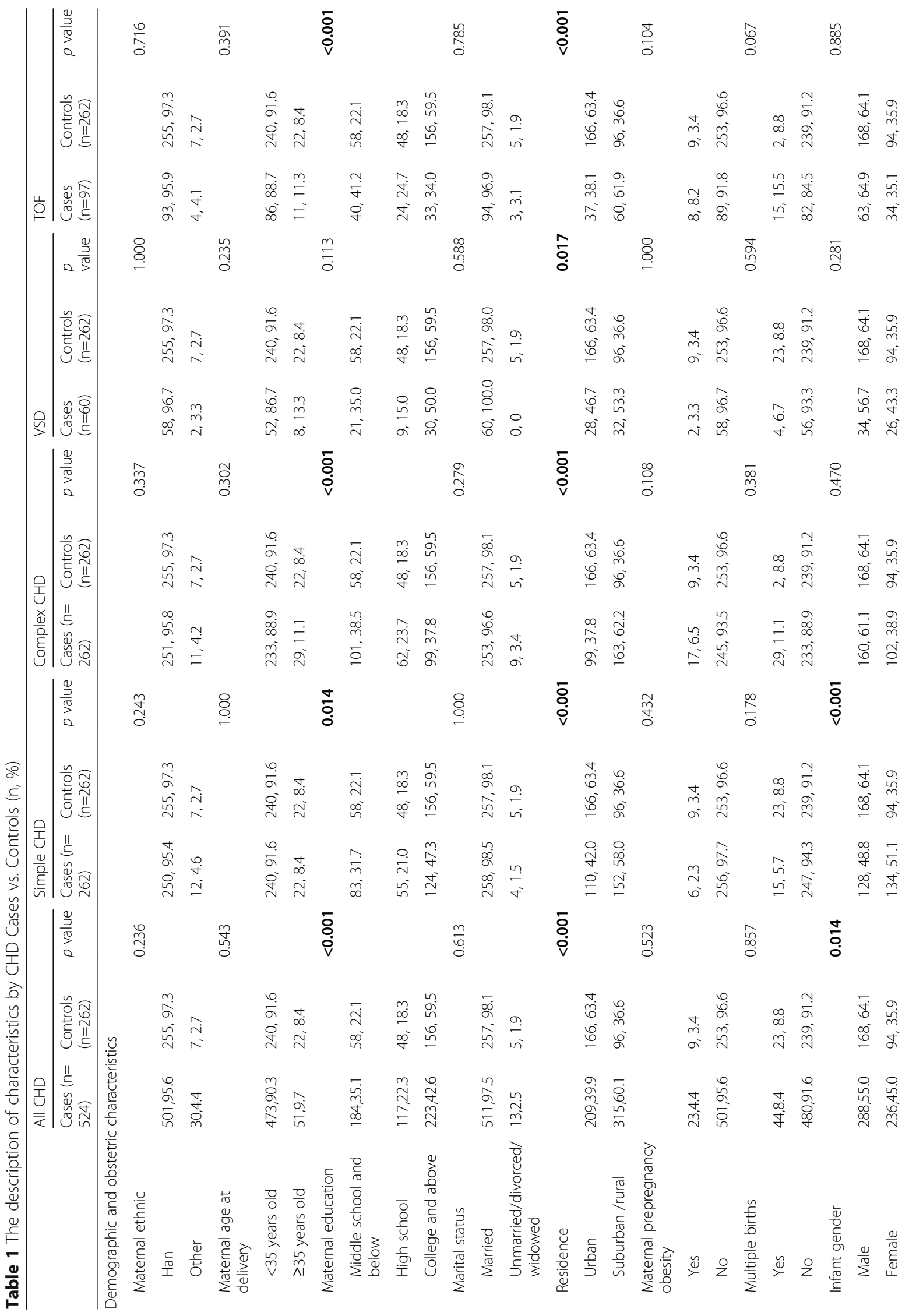


Xia et al. BMC Cardiovascular Disorders

(2019) 19:277

Page 6 of 13

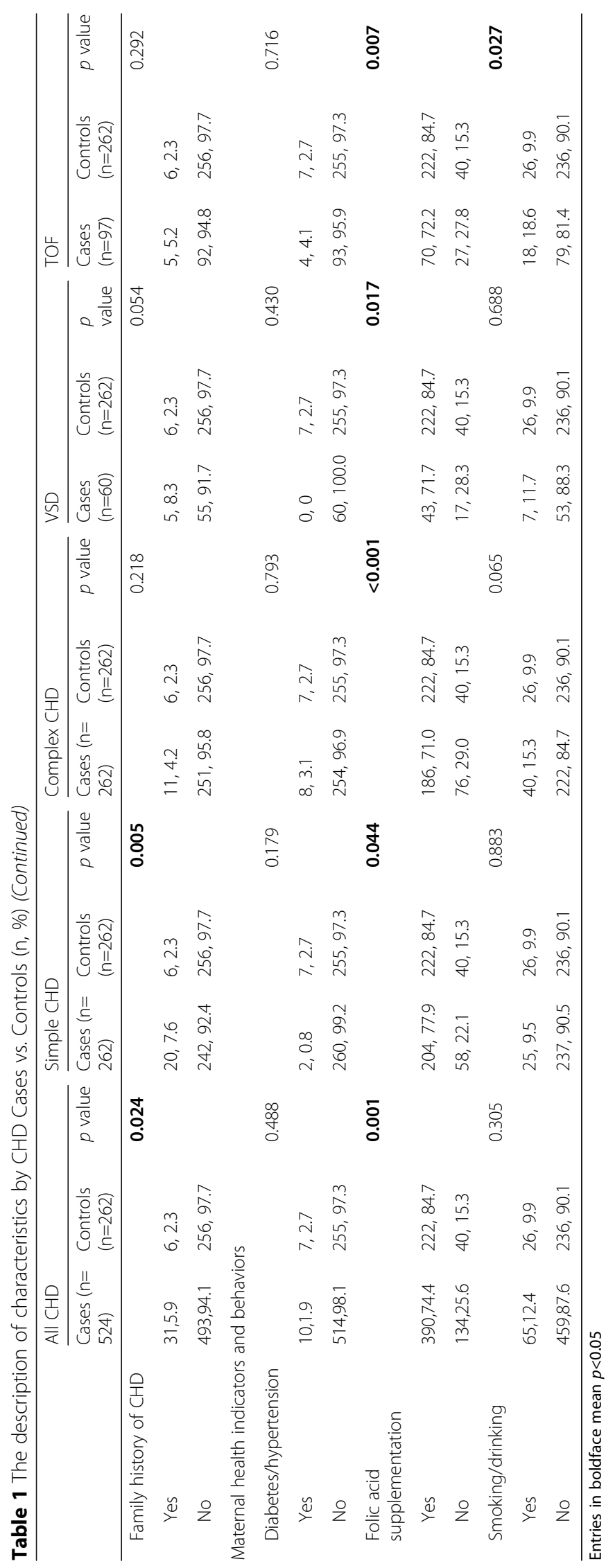


Table 2 Associations of maternal upper respiratory tract infection/ influenza during early pregnancy with congenital heart disease in offspring

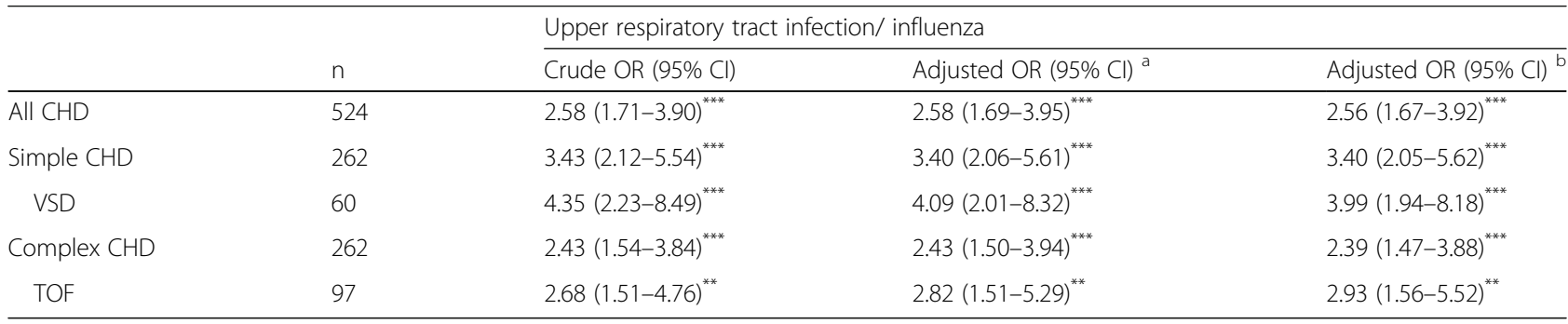

${ }^{a}$ Model I: adjusted for maternal ethic, maternal age at delivery, maternal education, marital status, residence, maternal prepregnancy obesity, multiple births, infant gender, and family history of CHD

${ }^{b}$ Model II: based on Model I, further adjusted for prepregnancy diabetes/hypertension, folic acid use, and smoking/drinking

${ }^{*} p$ value $<0.05 ;{ }^{* *} p$ value $<0.01 ;{ }^{* * *} p$ value $<0.001$

evidences and our findings. Our examinations were performed not only in all CHD as a whole group but also in subgroups of $\mathrm{CHD}$, simple $\mathrm{CHD}$ and complex $\mathrm{CHD}$. Meanwhile, for the first time, we particularly examined the associations in VSD and TOF, the most prevalent single type of simple and complex CHD, respectively. The very consistent associations were established among different groups of CHD and even single types of CHD in our case-control study. In the subsequent metaanalysis, except for TOF, the similar consistent associations still existed. Generally, our study proposed enriched evidence that maternal upper respiratory tract infection/influenza during early pregnancy was implicated in CHD, although more studies are still needed to verify the association in each single type of CHD.

A number of previous studies have explored the impact of upper respiratory tract infection or influenza on CHD [11-17, 21-25]. There is considerable overlap in etiology and symptomatology between upper respiratory tract infection and influenza [33], and the symptoms of

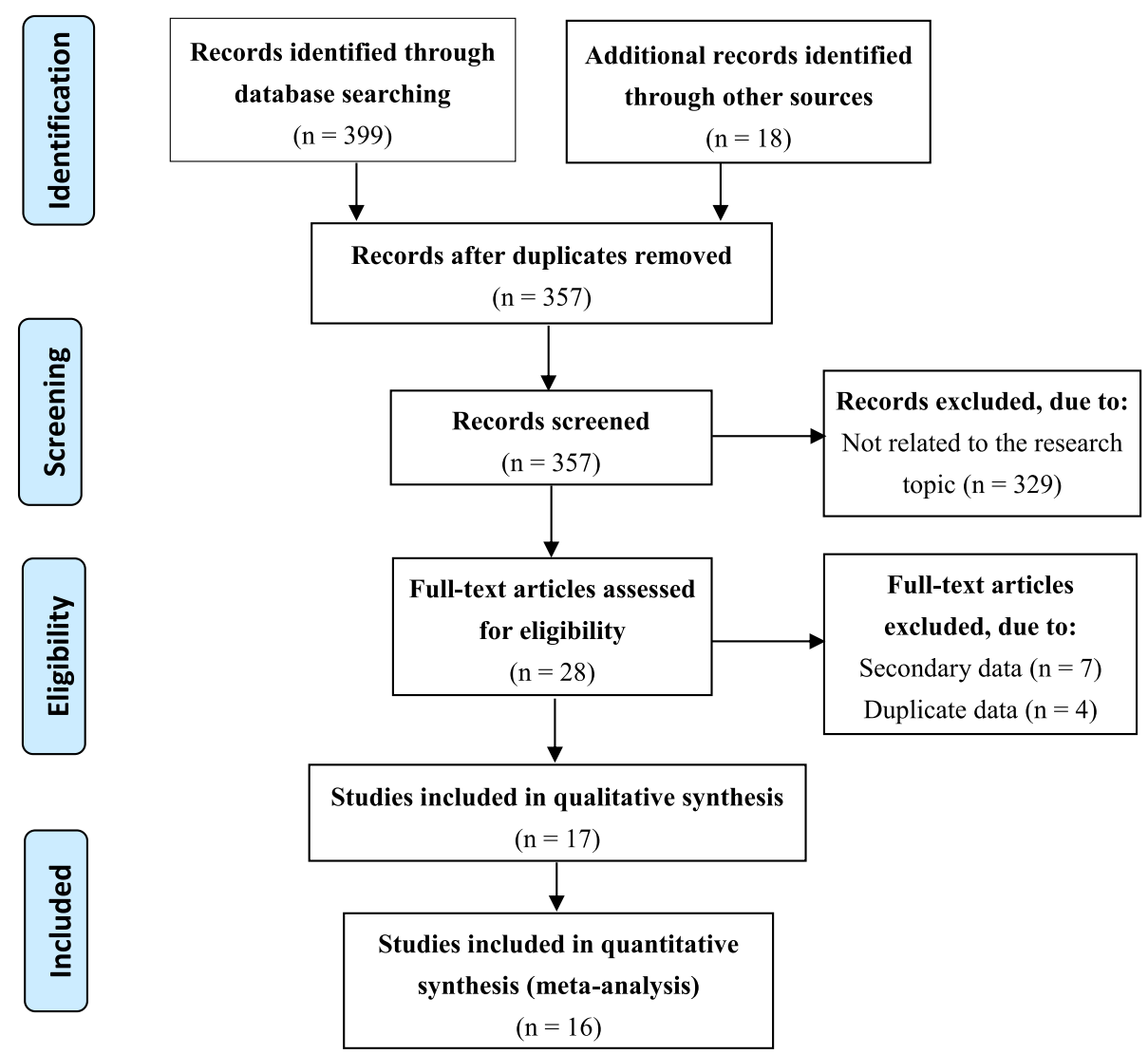

Fig. 1 Flow diagram of the selection process of the included/excluded studies in the meta-analysis 


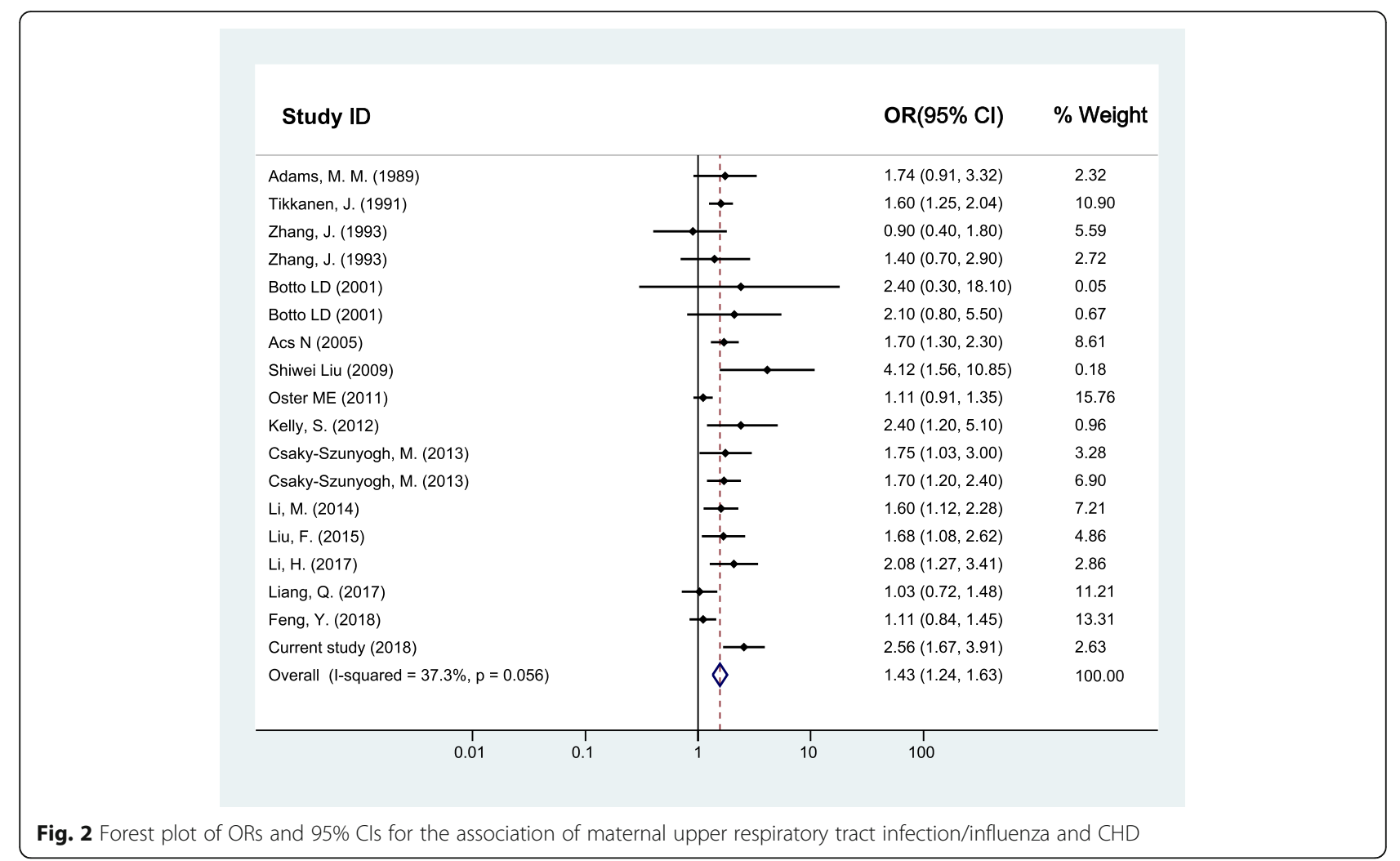

\section{Study ID}

Tikkanen, J. (1991)

Zhang, J. (1993)

Botto LD (2001)

Botto LD (2001)

Botto LD (2001)

Botto LD (2001)

Botto LD (2001)

Botto LD (2001)

Czeizel, A. E. (2008)

Czeizel, A. E. (2008)

Czeizel, A. E. (2008)

Oster ME (2011)

Oster ME (2011)

Oster ME (2011)

Csaky-Szunyogh, M. (2013)

Li, M. (2014)

Feng, Y. (2018)

Feng, Y. (2018)

Feng, Y. (2018)

Current study (2018)

Overall (I-squared $=26.6 \%, p=0.133$ )

\section{OR $(95 \% \mathrm{Cl}) \quad \%$ Weight}

$1.47(1.16,1.87) \quad 31.09$

$1.80(0.80,4.30) \quad 1.29$

$2.00(1.10,3.60) \quad 2.53$

$1.00(0.10,7.40) \quad 0.30$

$2.00(0.30,15.30) \quad 0.07$

$3.00(0.40,23.90) \quad 0.03$

$4.00(0.90,17.90) \quad 0.05$

$3.80(1.60,8.80) \quad 0.30$

$2.70(1.60,4.60) \quad 1.76$

$1.70(0.40,6.70) \quad 0.40$

$0.80(0.10,8.90) \quad 0.20$

$1.30(0.92,1.83) \quad 19.08$

$0.73(0.38,1.42) \quad 14.61$

$1.21(0.71,2.04) \quad 8.93$

$2.20(1.70,3.00) \quad 9.35$

$2.12(1.38,3.26) \quad 4.47$

$1.79(0.74,4.31) \quad 1.24$

$1.38(0.54,3.52) \quad 1.78$

$1.24(0.39,3.92) \quad 1.27$

$3.40(2.05,5.62) \quad 1.24$

$1.47(1.28,1.67) \quad 100.00$

100

Fig. 3 Forest plot of ORs and 95\% Cls for the association of maternal upper respiratory tract infection/influenza and simple CHD 


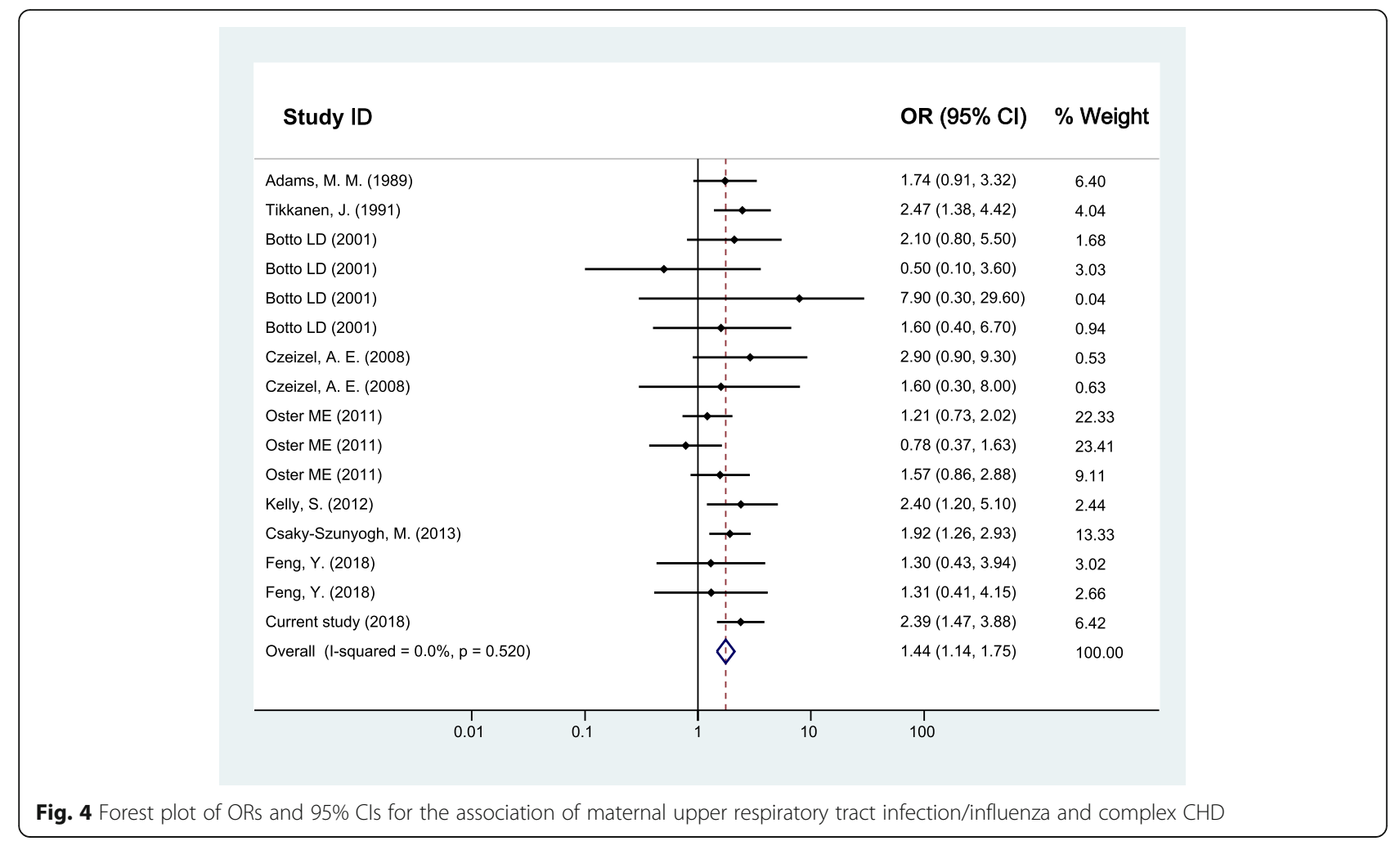

influenza are similar to the symptoms of upper respiratory tract infection, including fever, cough, sneezing, runny nose, sore throat, and etc. [33, 34]. For the common population and even medical professional, it is difficult to distinguish the two [34]. Therefore, we merged upper respiratory tract infection and influenza in the present study. A number of previous studies were conducted to explore the associations between upper respiratory tract infection or influenza and CHD [11-17, 21-25]. Usually, the studies observed the associations in

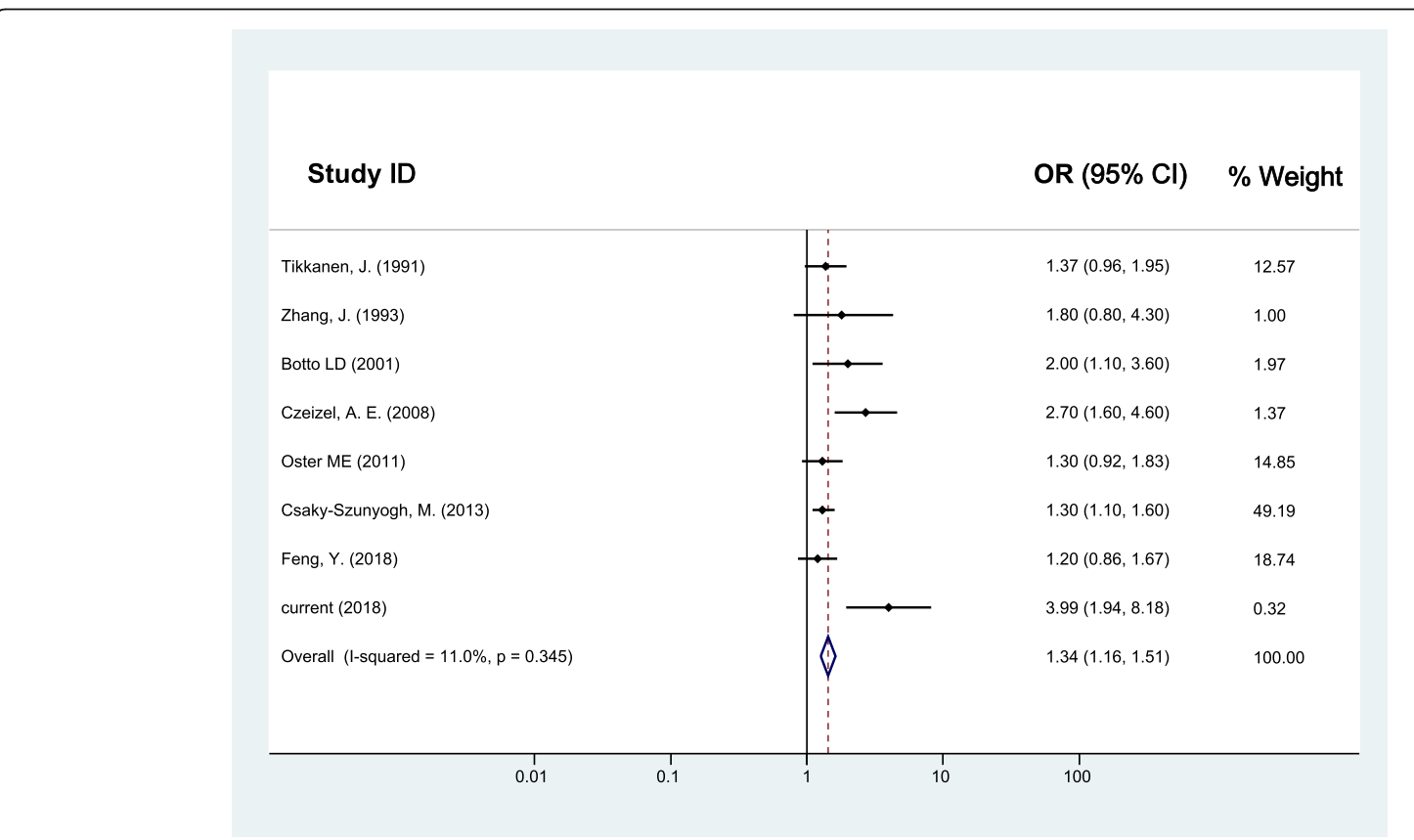

Fig. 5 Forest plot of ORs and 95\% Cls for the association of maternal upper respiratory tract infection/influenza and VSD 


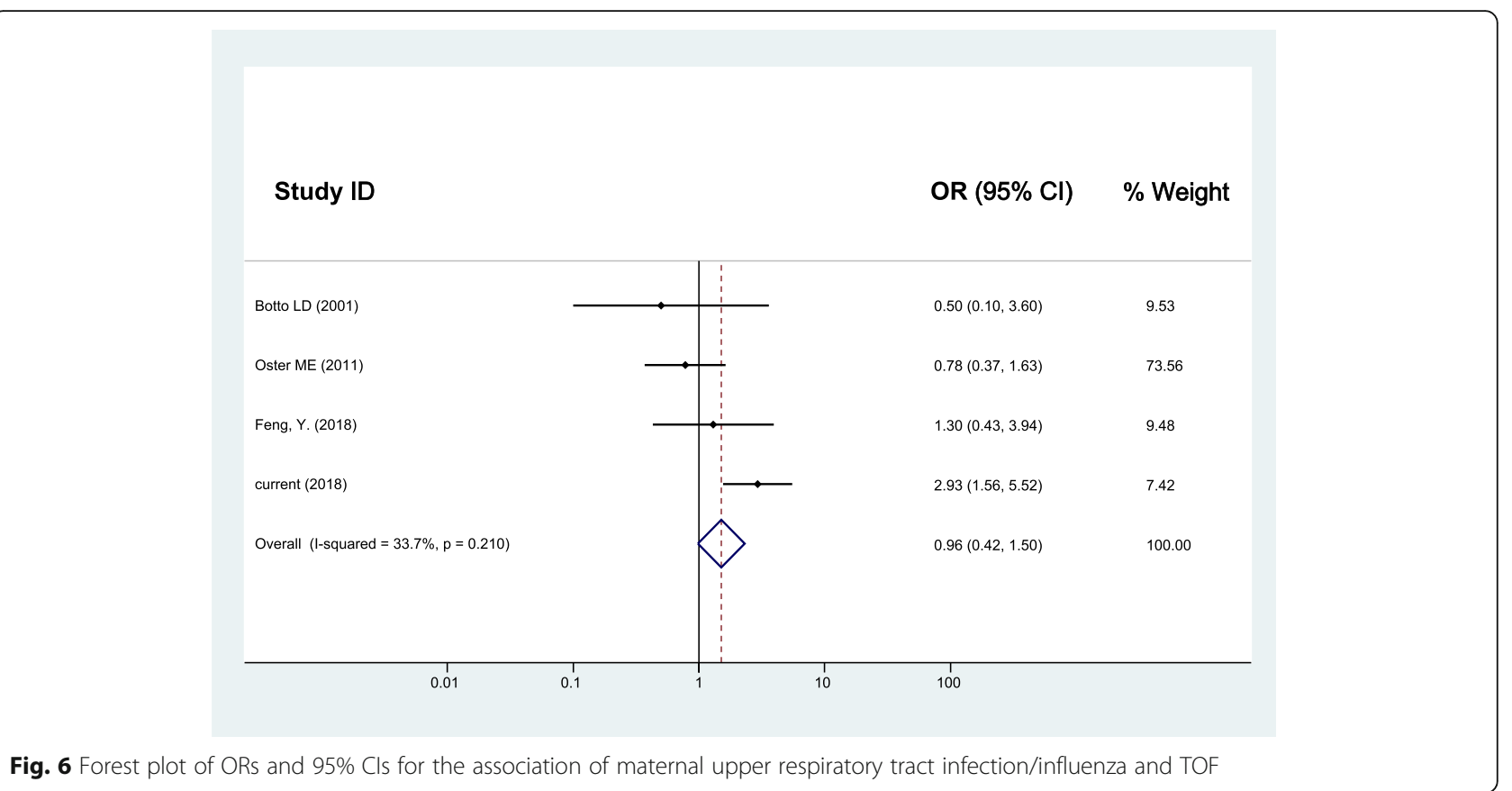

CHD as a whole, without considering the possible difference among different type of CHD [11-17, 21-25]. However, although the information is still somewhat limited, the available evidence demonstrated that the impacts of upper respiratory tract infection/influenza varied among subtypes of CHD [10, 18-20]. In our hospital-based case-control study, we set up two case groups as simple CHD and complex CHD, respectively. To the best of our knowledge, this is the first study exploring the associations of maternal upper respiratory tract infection/influenza with simple CHD and complex CHD separately. Furthermore, we also observed the associations in VSD and TOF, the most prevalent single type of simple and complex $\mathrm{CHD}$, respectively. Our case-control study showed that maternal upper respiratory tract infection/influenza could increase the risk of $\mathrm{CHD}$, and the adverse impacts were consistent either in subgroups of $\mathrm{CHD}$ or single types of CHD. The subsequent meta-analysis, except for TOF, overally confirmed our findings, which verified our findings and further enforced the evidence between upper respiratory tract infection/ influenza and CHD.

In the present study, upper respiratory tract infection/ influenza was found to be a risk factor for TOF. As far as we know, to date only three previous studies specifically examine the associations, however, by contrast to our study, none of them got the similar findings [13, 17 , 25]. The initial study to explore the correlation was a population-based case-control study conducted in Atlanta, the USA, in which 49 TOF cases vs. controls were analyzed, and among 49 cases, only one reported influenza exposure during pregnancy [13]. The limited case sample size may make the statistical model unstable and would affect the precision of the predictive value of the model. In addition, early pregnancy is the critical period for fetal cardiovascular development and, therefore, the susceptible window for CHD [6], however, the study collected information on influenza exposure without considering the stage of gestation. Similarly, another casecontrol study did not consider the susceptible period of influenza exposure during pregnancy for the risk of TOF [17]. A very recent study among 193 Chinese children with TOF examined the effects of influenza infection during different periods of pregnancy on the risk of TOF based on a 1:1 matched case-control study [25]. It seemed that maternal influenza exposure could increase the risk of TOF (OR ranged from 1.30 to 3.51 for influenza exposure during the first, second, and third trimester of pregnancy), although these results were not statistically significant. However, it is reasonable to deduce that the adverse effects would reach up to statistical significance with increasing sample size. Even so, it should be noticed that the value of OR in the second/ third trimester was greater than that of the first trimester, which suggested that recalling bias should be a major limitation to be taken into account when explaining the results. Presently, it was difficult to clearly explain why our results were different from these previous studies. We hope more studies will be performed in the future to verify these results.

European surveillance of congenital anomalies (EUROCAT) study recorded a total prevalence of major congenital anomalies of 23.9 per 1000 births for 2003-2007, in which that CHD was the most common non- 
chromosomal subgroup, at 6.5 per 1000 births [35]. $\mathrm{CHD}$ is the most common cause of major congenital anomalies, forming a major global health burden [1]. In the present study, we detected maternal upper respiratory tract infection/influenza during first trimester perinatal period as an independent risk factor of CHD, even after a meta-analysis. Although the mechanism by which maternal upper respiratory tract infection/influenza may result in CHD is still unclear, several potential explanations are feasible in theory to support the relationship. The majority of upper respiratory tract infection is caused by viruses and bacteria, and influenza is always caused by viruses [36]. It was revealed that upper respiratory tract infection viruses such as respiratory syncytial virus could cause a cytokine related immunological or inflammatory response [37, 38]. Upper respiratory tract infection/influenza is often accompanied by fever, the mechanism may be same to which fever influence CHD. Previous studies have suggested that both fever $[39,40]$ and influenza virus infection [41-43] could induce the apoptotic death of cells. Apoptosis is known to be involved in cardiac morphogenesis, and altered apoptosis has been suggested to cause birth defect [40]. Another possible pathway is a direct effect of influenza virus inducing cell death [44].

Several limitations should be acknowledged in interpreting the results. First, although the diagnosis of $\mathrm{CHD}$ is more accurate in hospital-based case-control studies than in population-based case-control studies, selection bias cannot be inevitable. To reduce the potential selection bias between cases and controls, propensity score matching was used, however, the method is usually used in clinical intervention study. Secondly, our exposure data were collected by retrospective maternal self-reported information, thus recall bias is a concern. Thirdly, there was publication bias in CHD group, and it may have some effect on the outcome of meta-analysis.

\section{Conclusions}

Although further studies are still needed to confirm the association of maternal upper respiratory tract infection/ influenza with CHD, especially some single type of CHD, our findings, to a large extent, solve the present controversy and indicate that maternal upper respiratory tract infection/influenza during the first trimester of pregnancy should be an independent risk factor for CHD. As a result, pregnant women should exercise properly, strengthen the mother's immunity to stay away from the source of infection.

\section{Supplementary information}

Supplementary information accompanies this paper at https://doi.org/10. 1186/s12872-019-1206-0.
Additional file 1. The search strategy in Embase.

Additional file 2: Table S1. The description of characteristics by CHD Cases vs. Controls (n, \%) ----Propensity-score-matched. Table S2. Associations of maternal upper respiratory tract infection/ influenza during early pregnancy with congenital heart disease in offspring--- after a propensity-score-matched analysis. Table S3. The results of Begg's test and Egger's linear regression test for the association of maternal upper respiratory tract infection/influenza and $\mathrm{CHD} /$ simple $\mathrm{CHD} /$ complex CHD. Figure S1. Forest plot of ORs and $95 \% \mathrm{Cls}$ for the association of maternal upper respiratory tract infection/influenza and CHD--- after a propensityscore-matched analysis. Figure S2. Forest plot of ORs and 95\% Cls for the association of maternal upper respiratory tract infection/influenza and simple CHD--- after a propensity-score-matched analysis. Figure S3. Forest plot of ORs and $95 \%$ Cls for the association of maternal upper respiratory tract infection/influenza and complex CHD--- after a propensityscore-matched analysis. Figure S4. Forest plot of ORs and 95\% Cls for the association of maternal upper respiratory tract infection/influenza and VSD--- after a propensity-score-matched analysis. Figure S5. Forest plot of ORs and $95 \% \mathrm{Cls}$ for the association of maternal upper respiratory tract infection/influenza and TOF--- after a propensity-score-matched analysis. Figure S6. Plot of sensitivity analysis for the association of maternal upper respiratory tract infection/influenza and CHD. Figure S7. Plot of sensitivity analysis for the association of maternal upper respiratory tract infection/influenza and simple CHD. Figure S8. Plot of sensitivity analysis for the association of maternal upper respiratory tract infection/influenza and complex CHD. Figure S9. Plot of sensitivity analysis for the association of maternal upper respiratory tract infection/influenza and VSD. Figure S10. Plot of sensitivity analysis for the association of maternal upper respiratory tract infection/influenza and TOF.

\section{Abbreviations}

CHD: Congenital heart disease; PBEQ: The parental behaviors and environmental exposure questionnaire; SPSS: The statistical package for the social sciences; TOF: Tetralogy of fallot; VSD: Ventricular septal defects

\section{Acknowledgements}

We would like to thank all the women who participated in this study, as well as doctors and nurses who assisted with recruitment for this research.

\section{Authors' contributions}

YQX: data analysis, profiling analysis, drafting the article; KNZ: acquisition of data; ADZ: help drafting the article, help summarize the data; IZZ: supervised the study; HFH: supervised the study; YLW: plan the study; SHL: plan the study, study design, revised the article. All authors have read and approved the final manuscript.

\section{Funding}

This research is supported by grants from National Natural Science Foundation of China $(81673183,81874266)$, key project from Shanghai Municipal Science and Technology Commission (18411951600), the Science and Technology Funds from Pudong New Area, Shanghai (PKJ2017-Y01), the Research Funds from Shanghai Jiao Tong University School of Medicine (20170509-1), the Scientific Research Development Funds from Xinhua Hospital, Shanghai Jiao Tong University School of Medicine (HX0251), and the Appropriate Technology Funds from the International Peace Maternal \& Child Health Hospital of Chinese welfare Institute (GFY5822). The funding sources had no role in the study design, data collection and analysis, decision to publish, or preparation of the manuscript.

\section{Availability of data and materials}

The database used for the study is not public but it is available from the corresponding author on reasonable request.

\section{Ethics approval and consent to participate}

The study conforms to the declaration of Helsinki, and the ethical application and consent procedure were approved by the Ethics Committee of

Shanghai Jiao Tong University School of Medicine (Approval number: SJUPN201717). The written informed consent from all of the participants or their guardians were obtained. 


\section{Consent for publication}

Not applicable.

\section{Competing interests}

The authors declare that they have no competing interests.

\section{Author details}

'School of Public Health, Shanghai Jiao Tong University, 227 South Chongqing Road, Huangpu District, Shanghai 200025, China. ${ }^{2}$ China Hospital Development Institute, Shanghai Jiao Tong University, Shanghai, China. ${ }^{3}$ Shanghai Children's Medical Center, Shanghai Jiao Tong University School of Medicine, Shanghai, China. ${ }^{4}$ Prenatal Diagnosis Department, International Peace Maternity \& Child Health Hospital, Shanghai Jiao Tong University School of Medicine, 910 Hengshan Road, Xuhui District, Shanghai 200030, China.

Received: 3 January 2019 Accepted: 24 September 2019 Published online: 02 December 2019

\section{References}

1. Van der Linde D, Konings EE, Slager MA, Witsenburg M, Helbing WA, Takkenberg JJ, Roos-Hesselink JW. Birth prevalence of congenital heart disease worldwide: a systematic review and meta-analysis. J Am Coll Cardiol. 2011:58(21):2241-7.

2. Zhang Y, Riehle-Colarusso T, Correa A, Li S, Feng X, Gindler J, Lin H, Webb C, Li W, Trines J, et al. Observed prevalence of congenital heart defects from a surveillance study in China. J Ultrasound Med. 2011;30(7):989-95.

3. Roncancio CP, Misnaza SP, Pena IC, Prieto FE, Cannon MJ, Valencia D. Trends and characteristics of fetal and neonatal mortality due to congenital anomalies, Colombia 1999-2008. J Matern Fetal Neonatal Med. 2018;31(13): $1748-55$

4. Qu Y, Liu X, Zhuang J, Chen G, Mai J, Guo X, Ou Y, Chen J, Gong W, Gao X, et al. Incidence of congenital heart disease: the 9-year experience of the Guangdong registry of congenital heart disease, China. PLoS One. 2016; 11(7):e0159257.

5. Ferencz C, Boughman JA, Neill CA, Brenner Jl, Perry LW. Congenital cardiovascular malformations: questions on inheritance. BaltimoreWashington infant study group. J Am Coll Cardiol. 1989;14(3):756-63.

6. Gorini F, Chiappa E, Gargani L, Picano E. Potential effects of environmenta chemical contamination in congenital heart disease. Pediatr Cardiol. 2014; 35(4):559-68.

7. Neuzil KM, Reed GW, Mitchel EF, Simonsen L, Griffin MR. Impact of influenza on acute cardiopulmonary hospitalizations in pregnant women. Am J Epidemiol. 1998:148(11):1094-102

8. Dodds L, McNeil SA, Fell DB, Allen VM, Coombs A, Scott J, MacDonald N. Impact of influenza exposure on rates of hospital admissions and physician visits because of respiratory illness among pregnant women. Cmaj. 2007 176(4):463-8.

9. Luteijn JM, Brown MJ, Dolk H. Influenza and congenital anomalies: a systematic review and meta-analysis. Hum Reprod. 2014;29(4):809-23.

10. Adams MM, Mulinare J, Dooley K. Risk factors for conotruncal cardiac defects in Atlanta. J Am Coll Cardiol. 1989;14(2):432-42.

11. Tikkanen J, Heinonen OP. Maternal hyperthermia during pregnancy and cardiovascular malformations in the offspring. Eur J Epidemiol. 1991;7(6):628-35.

12. Zhang J, Cai WW. Association of the common cold in the first trimester of pregnancy with birth defects. Pediatrics. 1993;92(4):559-63.

13. Botto LD, Lynberg MC, Erickson JD. Congenital heart defects, maternal febrile illness, and multivitamin use: a population-based study. Epidemiology. 2001:12(5):485-90.

14. Acs N, Banhidy F, Puho E, Czeizel AE. Maternal influenza during pregnancy and risk of congenital abnormalities in offspring. Birth Defects Res A Clin Mol Teratol. 2005;73(12):989-96

15. Czeizel AE, Puho EH, Acs N, Banhidy F. Use of specified critical periods of different congenital abnormalities instead of the first trimester concept. Birth Defects Res A Clin Mol Teratol. 2008;82(3):139-46.

16. Liu S, Liu J, Tang J, Ji J, Chen J, Liu C. Environmental risk factors for congenital heart disease in the Shandong peninsula, China: a hospital-based case-control study. J Epidemiol. 2009;19(3):122-30.

17. Oster ME, Riehle-Colarusso T, Alverson CJ, Correa A. Associations between maternal fever and influenza and congenital heart defects. J Pediatr. 2011; 158(6):990-5.
18. Kelly S, Kuehl K, Loffredo C. Tricuspid atresia: an epidemiologic investigation of live-born cases in the Baltimore-Washington area. Am J Epidemiol. 2012;175:S59

19. Csaky-Szunyogh M, Vereczkey A, Kosa Z, Gerencser B, Czeizel AE. Risk and protective factors in the origin of conotruncal defects of heart--a population-based case-control study. Am J Med Genet A. 2013;161a(10): 2444-52.

20. Csaky-Szunyogh M, Vereczkey A, Kosa Z, Urban R, Czeizel AE. Association of maternal diseases during pregnancy with the risk of single ventricular septa defects in the offspring--a population-based case-control study. J Matern Fetal Neonatal Med. 2013:26(8):738-47.

21. Li M, Liu Z, Lin Y, Chen X, Li S, You F, Deng Y, Li N, Wang Y, Zhang Y, et al. Maternal influenza-like illness, medication use during pregnancy and risk of congenital heart defects in offspring. J Matern Fetal Neonatal Med. 2014; 27(8):807-11.

22. Liu F, Yang YN, Xie X, Li XM, Ma X, Fu ZY, Chen BD, Huang Y, Shan CF, Ma $Y T$, et al. Prevalence of congenital heart disease in Xinjiang multi-ethnic region of China. PLoS One. 2015;10(8):e0133961.

23. Li H, Luo M, Zheng J, Luo J, Zeng R, Feng N, Du Q, Fang J. An artificial neural network prediction model of congenital heart disease based on risk factors: a hospital-based case-control study. Medicine (Baltimore). 2017;96(6):e6090

24. Liang Q, Gong W, Zheng D, Zhong R, Wen Y, Wang X. The influence of maternal exposure history to virus and medicine during pregnancy on congenital heart defects of fetus. Environ Sci Pollut Res Int. 2017;24(6):5628-32

25. Feng $Y$, Cai J, Tong X, Chen R, Zhu Y, Xu B, Mo X. Non-inheritable risk factors during pregnancy for congenital heart defects in offspring: a matched case-control study. Int J Cardiol. 2018:264:45-52.

26. Zhao A, Zhao K, Xia Y, Yin Y, Zhu J, Hong H, Li S. Exploring associations of maternal sleep during periconceptional period with congenital heart disease in offspring. Birth Defects Res. 2019;111(13):920-31.

27. Wu MH, Chen HC, Lu CW, Wang JK, Huang SC, Huang SK. Prevalence of congenital heart disease at live birth in Taiwan. J Pediatr. 2010; 156(5):782-5.

28. Yeh SJ, Chen HC, Lu CW, Wang JK, Huang LM, Huang SC, Huang SK, Wu $\mathrm{MH}$. National database study of survival of pediatric congenital heart disease patients in Taiwan. J Formos Med Assoc. 2015;114(2):159-63.

29. Higgins JPT, Thompson SG. Quantifying heterogeneity in a meta-analysis. Stat Med. 2002;21(11):1539-58.

30. Dekkers OM, Vandenbroucke JP, Cevallos M, Renehan AG, Altman DG, Egger M. COSMOS-E: guidance on conducting systematic reviews and metaanalyses of observational studies of etiology. PLoS Med. 2019;16(2): e1002742.

31. Duval S, Tweedie R. Trim and fill: a simple funnel-plot-based method of testing and adjusting for publication bias in meta-analysis. Biometrics. 2000; 56(2):455-63.

32. Eng C, Kramer CK, Zinman B, Retnakaran R. Glucagon-like peptide-1 receptor agonist and basal insulin combination treatment for the management of type 2 diabetes: a systematic review and meta-analysis. Lancet. 2014;384(9961):2228-34.

33. Eccles R. Mechanisms of symptoms of the common cold and influenza. $\mathrm{Br}$ Hosp Med (Lond). 2007;68(2):71-5.

34. Eccles R. Understanding the symptoms of the common cold and influenza. Lancet Infect Dis. 2005;5(11):718-25.

35. Dolk H, Loane M, Garne $\mathrm{E}$. The prevalence of congenital anomalies in Europe. Adv Exp Med Biol. 2010;686:349-64.

36. Larson E, Ferng YH, Wong J, Alvarez-Cid M, Barrett A, Gonzalez MJ, Wang S, Morse SS. Knowledge and misconceptions regarding upper respiratory infections and influenza among urban Hispanic households: need for targeted messaging. J Immigr Minor Health. 2009;11(2):71-82.

37. Sung RY, Hui SH, Wong CK, Lam CW, Yin J. A comparison of cytokine responses in respiratory syncytial virus and influenza a infections in infants. Eur J Pediatr. 2001;160(2):117-22.

38. Matsuse $H_{1}$, Tsuchida T, Fukahori S, Kawano T, Tomari S, Matsuo N, Nishino T, Fukushima C, Kohno S. Differential airway inflammatory responses in asthma exacerbations induced by respiratory syncytial virus and influenza virus a. Int Arch Allergy Immunol. 2013;161(4):378-82.

39. Mirkes PE, Cornel LM, Park HW, Cunningham ML. Induction of thermotolerance in early postimplantation rat embryos is associated with increased resistance to hyperthermia-induced apoptosis. Teratology. 1997;56(3):210-9. 
40. Edwards MJ. Apoptosis, the heat shock response, hyperthermia, birth defects, disease and cancer. Where are the common links? Cell Stress Chaperones. 1998;3(4):213-20.

41. Takizawa T, Matsukawa S, Higuchi Y, Nakamura S, Nakanishi Y, Fukuda R. Induction of programmed cell death (apoptosis) by influenza virus infection in tissue culture cells. J Gen Virol. 1993;74(Pt 11):2347-55.

42. Fesq $\mathrm{H}$, Bacher M, Nain M, Gemsa D. Programmed cell death (apoptosis) in human monocytes infected by influenza a virus. Immunobiology. 1994; 190(1-2):175-82.

43. Hinshaw VS, Olsen CW, Dybdahl-Sissoko N, Evans D. Apoptosis: a mechanism of cell killing by influenza a and B viruses. J Virol. 1994;68(6): 3667-73.

44. Takizawa T, Ohashi K, Nakanishi Y. Possible involvement of double-stranded RNA-activated protein kinase in cell death by influenza virus infection. J Virol. 1996;70(11):8128-32.

\section{Publisher's Note}

Springer Nature remains neutral with regard to jurisdictional claims in published maps and institutional affiliations.

Ready to submit your research? Choose BMC and benefit from:

- fast, convenient online submission

- thorough peer review by experienced researchers in your field

- rapid publication on acceptance

- support for research data, including large and complex data types

- gold Open Access which fosters wider collaboration and increased citations

- maximum visibility for your research: over $100 \mathrm{M}$ website views per year

At BMC, research is always in progress.

Learn more biomedcentral.com/submissions 